\title{
A QoS-oriented distributed routing protocol for hybrid wireless networks
}

\author{
Mathumathi $^{1}$, Manjusha.T.M ${ }^{2}$ \\ Assistant Prof., Dept., of Computer Science, Sree Narayana Guru College of Arts \& Science, Coimbatore, TamilNadu ${ }^{1}$ \\ M.Phil. Scholar, Dept., of Computer Science, Sree Narayana Guru College of Arts \& Science, Coimbatore, TamilNadu ${ }^{2}$
}

\begin{abstract}
With the increasing level of wireless communication in today's environment, people often required QOS for sharing their data between the nodes. For affording QOS to the user, many researchers proposed a very few methods to provide QoS guaranteed routing for hybrid networks, they strive to improve the network capacity and reliability but they evade constrain in QOS. For this problem our main objective of this paper is to improve the QOS and efficiency of routing approach with constrains over hybrid wireless data streaming using QOS_DARP protocol and MAR. This aims to develop the QOS based reliable architecture against the hybrid wireless routing issues. The system also aims at providing both proactive and reactive solutions for effective routing. The goal of this paper is to providing efficient dynamic routing management to deal the challenges of data transmission and data streaming in hybrid mobile environments.
\end{abstract}

Keywords: Hybrid Wireless Network, QoS, QOS_DARP and MAP protocol.

\section{INTRODUCTION}

Hybrid Wireless Communication (HWC) is one of the In the above figure which shows the integration of wired popular among the people for sharing the resources among and wireless networks, uses base stations to avoid over the networks. This Hybrid approach is based on the whelming burden of relaying packets between source and combination of sensor, mobile ad hoc, vehicular ad hoc destination if required. Presenting robust multicast routing network for sharing the resources in the distributed in those dynamic network environments is a significant network. With the use of this communication people often challenge for sustaining these applications [3, 4]. In a few needs the QOS while transferring the data [1]. In MOBILE wireless multicast applications, the source and ad hoc networks (MANETs) have expanded a great deal of intermediary nodes are mobile for transferring the data, concentration since of its considerable advantages brought but the multicast recipients' locations are fixed and known about by multihop, infrastructure-less transmission. Due to they also sent the packet in the same intermediate node. the error prone wireless channel and the dynamic network Conversely, in other wireless multicast applications, all topology, consistent data delivery in MANETs, mainly in nodes, with the multicast destinations, are mobile. In this this challenge environment with high mobility remains an paper, in order to support any type of multicast service to issue. In the real world, a numerous applications need data the target devices, the source nodes must recognize the delivery to multiple destination nodes which is defined as locations of the multicast target or destination nodes. This multicasting, where the access of multicast routing is an can be affording by a service discovery protocol that sits ideal approach to manage and reduce network traffic and outside the routing protocol, revising the source with the cost of the network including bandwidth. Oftentimes these current location of the sink nodes [5]. Moreover, the services are required over extremely in dynamic networks, routing protocol can take for granted knowledge of the which is Hybrid approach of above combination of ad hoc sinks' locations. We can utilize this knowledge to design a network. These networks are dynamic because of the distributed QOS routing protocol. In this paper, the system mobility of the nodes in the network and/or the random improves the work on a QOS-Based scheduling protocol sleep/awake cycles that are often utilized to minimize which is called as QAR [6,7].

energy dissipation of the devices [2]

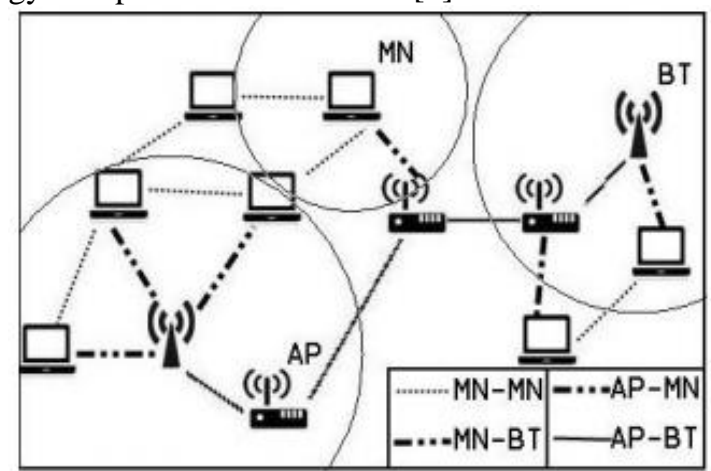

Fig 1: Hybrid Wireless Communication
The proposed system is an effective QOS based link selection in hybrid wireless networks (QOS_DARP) which is a distributed QOS protocol where the system performs packet security, packet routing, multiple routes which is commonly for splitting packets and the medium access of individual nodes relies solely on the location information of multicast destination nodes. The proposed system embraces a list of the multicast members' locations included in the packet header, which avoid the overhead of building and preserving a multicast tree at intermediate of the sensor nodes, since all the essential information for routing the packet is included within the packet header. 


\section{LITERATURE REVIEW}

In literature some authors proposed a resource provision method in hybrid networks modeled by and mobile WiMax to provide service with high reliability.

IntServ is a stateful model that uses resource reservation for individual flow, and uses admission control [8] and a scheduler to maintain the QoS of traffic flows. In difference, DiffServ is a stateless form which uses coarse grained class-based apparatus for traffic management. Queuing scheduling algorithms have been proposed for DiffServ to further minimize packet droppings and bandwidth consumption [9]. Stoica et al. [10] proposed a dynamic packet service (DPS) model to provide unicast IntServ-guaranteed service and Diffservlike scalability.

In existing approaches for providing guaranteed services in the infrastructure networks are based on two models: integrated services (IntServ) and differentiated service (DiffServ).Very few methods have been proposed to provide QoSguaranteed routing for hybrid networks. Most of the routing protocols only try to improve the network capacity and reliability to indirectly provide QoS service but bypass the constraints in QoS routing that require the protocols to provide guaranteed service. Jiang et al. [11] proposed a resource provision method in hybrid networks modeled by IEEE802.16e and mobile WiMax to provide service with high reliability. Ibrahim et al. [12] and Bletasa et al. [13] also tried to select "best" relay that has the maximum instantaneous value of a metric which can achieve higher bandwidth efficiency for data transmission. $\mathrm{Ng}$ and $\mathrm{Yu}$ [14] considered cooperative networks that use physical layer relaying strategies, which take advantage of the broadcast nature of wireless channels and allow the destination to cooperatively "combine" signals sent by both the source and the relay to restore the original signal. Cai et al. [15] proposed a semidistributed relaying algorithm to jointly optimize relay selection and power allotment of the system. Wei et al. [16] proposed to utilize the first-order finite state Markov channels to approximate the time variations of the average received signal-to-noise ratio (SNR) for the packet transmission and use the adaptive modulation and coding scheme to achieve high spectral efficiency and presented a framework of link capacity analysis for optimal transmission over uplink transmission in multi hop cellular networks. Weiet al. [17] proposed a two-hop packet forwarding mechanism, in which the source node adaptively chooses direct transmission and forward transmission to base stations. Unlike the above works, QOD aims to provide QoS guaranteed routing. QOD fully takes advantage of the widely deployed APs, and novelly considers the packet routing problem as a resource scheduling problem between nodes and APs.

RAP [18] and SPEED [19] give a high delivering priority to the packets with longer distance/delay to the destination. However, both methods require each sensor to know its own location, thus they are not suitable for a highly dynamic environment and proposed to improve routing reliability by multipath routing. Nevertheless, the surplus transmission of the packets may lead to high power consumption.

\section{PROBLEM DEFINITION}

The problem in the existing system is defined in the terms of reliable data delivery of data transmission in highly dynamic mobile hybrid networks [20]. Continuously changing network topology makes conventional wireless routing protocols incapable of providing satisfactory performance in the data transaction environment. In the face of frequent link break because of the node mobility, major data packets would either get lost, or knowledge long latency before reinstatement of connectivity. In Dynamic network topology the problems were

$\wp \quad$ Frequent link break

$\wp \quad$ Latency and Data loss

$\wp \quad$ High mobility

Hybrid wireless network topologies usually expose high link density. Low-end commodity switches are commonly used in most HWNs designs for economic and scalability considerations. Mobile destinations (nodes) create a challenging problem for QOS protocols. Several protocols dealt the above problem by applying region based throughput and delay [21, 22]. But those systems failed to deal with the high mobility in HWN environment.

\section{PROPOSED WORK}

With the improvement of the wireless network for solving the QOS issues, here we prefer the approach of QOS_DARP. A HWN is integrated with MANETs and infrastructure wireless networks have proven to be a better network structure for the next generation networks. However, little effort has been devoted to supporting QoS routing in hybrid networks. Direct adoption of the QoS routing techniques in MANETs into hybrid networks inherits their drawbacks. For this issue this paper, we propose a QoS oriented distributed routing protocol (QOD) for hybrid networks to provide QoS services in a highly dynamic scenario. Taking advantage of the unique features of hybrid networks, i.e., any cast transmission and short transmission hops, QOD transforms the packet routing problem to a packet scheduling problem. In QOD, a source node directly transmits packets to an AP if the direct transmission can guarantee the QoS of the traffic. Or else, the source node schedules the packets to a number of qualified neighbor nodes. Specifically, QOD incorporates with the algorithms are, Neighbor selection algorithm for the guaranteed QoS, it chooses qualified neighbors for packet forwarding.

Packet scheduling algorithm for schedules the packet transmission to further reduce the packet transmission time and we use the resizing algorithm, it is used to resizing the packets and assigns smaller packets to nodes with faster mobility to guarantee the routing QoS in a highly mobile environment. The traffic elimination algorithm is used for increase the transmission throughput. And use the Deadline-based forwarding methods for achieves fairness in packet forwarding scheduling when some packets are not scheduling feasible. Experimental results show that QOD can achieve high mobility-flexibility, scalability, and disputation reduction. 


\section{PROPOSED ARCHITECTURE}

The proposed distributed enhanced routing approaches which will reduce service disruption and minimize the worst-case maximal link utilization after link failures. Every link will be analyzed and updated in the routing table using the Q-ORP frequently. Below figure shows our proposed architecture.

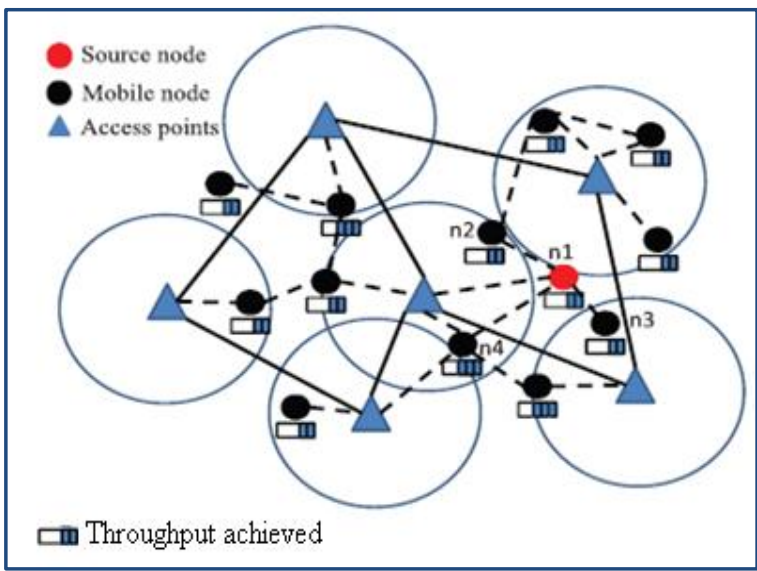

Fig 2: Our Proposed Architecture of HWC

Our proposed protocol adopts the resource reservation based QOS routing scheme. In this proposal a QOSOriented Distributed optimal routing protocol (Q-ORP) to enhance the QoS support capability of hybrid networks.

The proposed protocol can provide high QoS performance in terms of transparency, delay in transmission, delay in propagation, queuing delay, mobility-resilience, traffic reduction, and scalability.

\section{Proposed algorithm:}

\section{Nearest Neighbor Finding Algorithm}

\section{Algorithm: Distance Measurement}

Input: Objects positions $(\mathrm{o} 1, \mathrm{o} 2)$

Output: Distance (in points)

Step1: Identify object A

Step2: Identify Object B

Step3: Identify position of object $1 \mathrm{x}$ and y $\mathrm{O} 1(\mathrm{x} 1$, y1)

Step 4: Identify position of object $2 \mathrm{x}$ and $\mathrm{y}$ $\mathrm{O} 1(\mathrm{x} 2, \mathrm{y} 2)$

Step5: difference between (x1-x2) and (y1-y2)

Step6: Return value

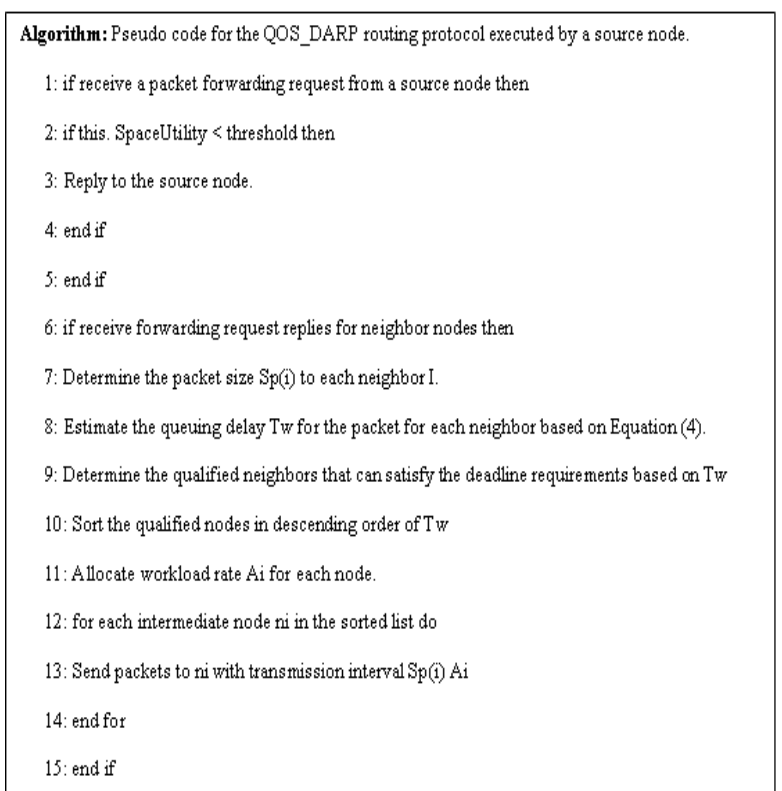

Finally the proposed protocol Q-ORP works with the above QOS metrics such as follows.

$\wp \quad$ With the use of neighbor selection algorithm it solves the transmission delay problems.

$\wp \quad$ A distributed packet scheduling algorithm to further reduce transmission delay, propagation and queuing delay

$\wp \quad$ A mobility-based segment resizing algorithm that adaptively adjusts segment size according to node mobility in order to reduce transmission time,

$\wp \quad$ Increase the transmission throughput use of redundant elimination algorithm.

$\wp$ A data redundancy elimination-based transmission algorithm to eliminate the redundant data to further improve the transmission QoS.

\section{EXPERIMENTAL RESULT}

The experimental result shows the difference between the existing and the proposed architecture for providing the better result for the QOS in Hybrid network. The system creates $\mathrm{N}$ number of nodes and performs the QOS oriented routing using the QOS_DARP.

The result analysis gives the comparative analysis of existing and proposed system in terms of Qos. The charts show that the proposed system achieved the improved QoS.

Evaluation result: The result analysis gives the comparative analysis of existing and proposed system in terms of Qos.

Packet Delivery Ratio (PDR): The ratio of number of packets send from source and number of packets reach the destination. The ratio of the number of packets received and the number of packets expected to be received. Then the ratio is the total number of received packets over the multiplication of the group size and the number of originated packets. The packet delivery ratio is calculated as follows: 
International Journal of Advanced Research in Computer and Communication Engineering Vol. 4, Issue 1, January 2015

PDR $=($ No of packets Received $/$ No of packets send $) * 100$

\begin{tabular}{|ll|}
\hline QOD Protocol & $\begin{array}{l}\text { QOS_DARP with } \\
\text { MAR }\end{array}$ \\
\hline $\mathbf{8 9}$ & 97 \\
\hline $\mathbf{9 2}$ & 98 \\
\hline $\mathbf{9 4}$ & 99 \\
\hline $\mathbf{9 5}$ & 99 \\
\hline
\end{tabular}

Table 1: Packet Delivery Ratio

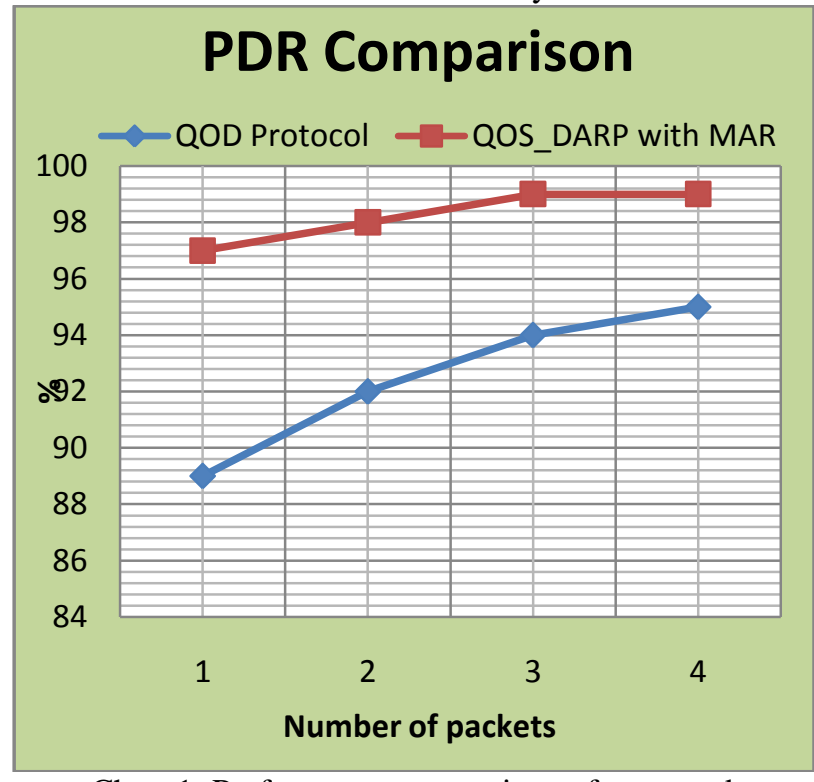

Chart 1: Performance comparison of proposed

QOS_DARP using MAR with existing QOD approach Delivery Time: The proposed system schedules the message in optimal path and the message reach the destination in any of the available best nodes and paths .Thus Delivery time of proposed system increases when compared to the existing system. Delivery time is calculated as follows:

Deliverv Time $=$ Receivino time - Sendino time

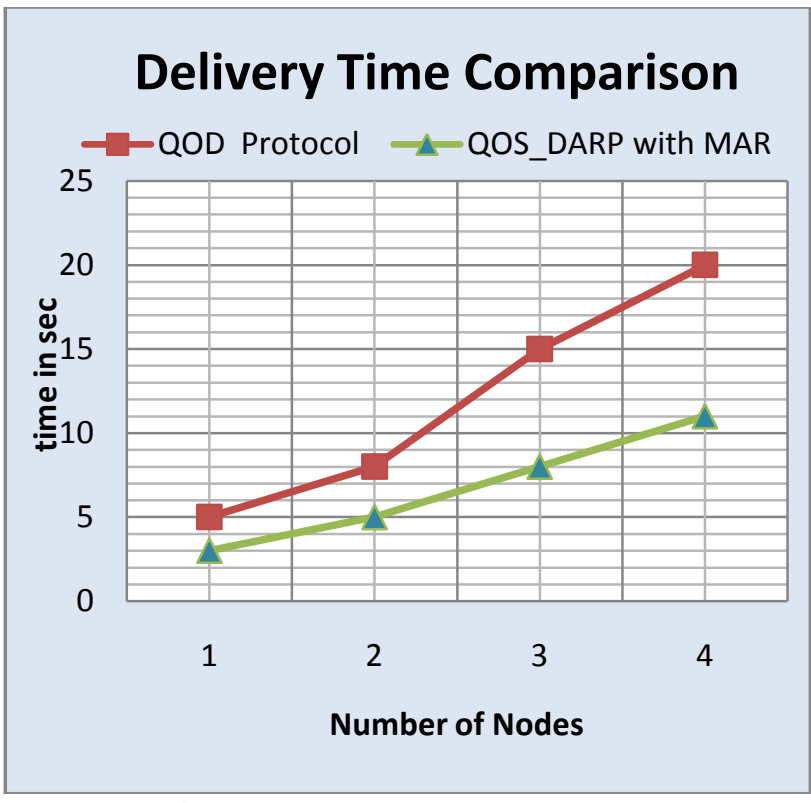

Chart 2: Delivery Time Comparison
Bandwidth Utilization: The bandwidth refers to the bandwidth or energy consumption of the link. The proposed system spread the message in the available virtual nodes and paths by proper utilization of bandwidth in each path with the use of VDVH.

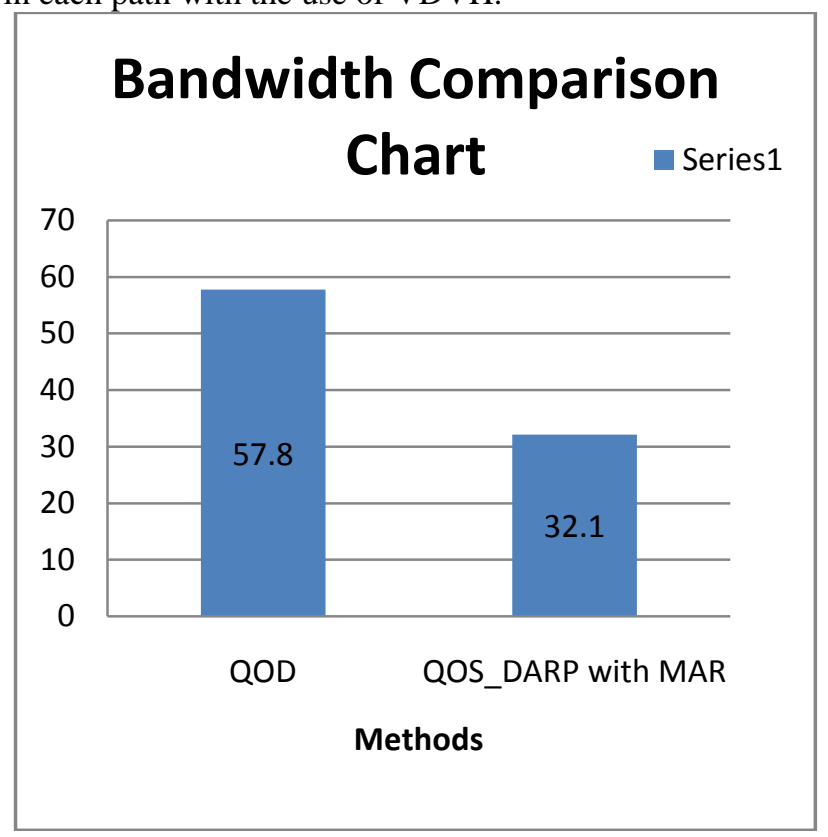

Chart 3: Bandwidth comparison of proposed QOS_DARP using MAR with existing QOD approach

\section{CONCLUSION \& FUTURE ENHANCEMENT}

This paper proposed an efficient secure distributed QOS protocol that addresses some issues specific to Hybrid wireless networks which are communication delay, cost, mobility, and link unreliability. Nodes affix the details about the forwarding nodes in the packet header. This also creates a virtual node for effective data transmission. The system implements an appropriate scheme which is named as MAR for mobility analysis during transmission. This helps to find closest neighbor already in the group therefore reducing the cost of join requests broadcast and reducing the communication and computation cost incurred by the source. In future the thesis will be enhanced by applying effective algorithms and better protocol with other parameter considerations. The Qos metrics will be measured more in future. The concentration on selective congestion attacks by applying effective traffic and node monitoring techniques. The priority based traffic allocation with effective technique will be considered more in future.

\section{REFERENCES}

[1] Shah R, Rabaey J. Energy aware routing for low energy ad hoc sensor networks. Proceedings of IEEE WCNC'02, Orlando, FL, March, 2002; 350-355.

[2] Ye Ming Lu, Vincent W.S. Wong, An energy efficient multipath routing protocol for wireless sensor networks, International Journal of Communication System 20 (7) (2007) 747-766.

[3] T. Reddy, I. Karthigeyan, B. Manoj, and C. Murthy, "Quality of Service Provisioning in Ad Hoc Wireless Networks: A Survey of Issues and Solutions," Ad Hoc Networks, vol. 4, no. 1, pp. 83-124, 2006.

[4] I. Jawhar and J. Wu, "Quality of Service Routing in Mobile Ad Hoc Networks,” Network Theory and Applications, Springer, 2004. 
[5] X. Du, "QoS Routing Based on Multi-Class Nodes for Mobile Ad Hoc Networks," Ad Hoc Networks, vol. 2, pp. 241-254, 2004.

[6] M. Conti, E. Gregori, and G. Maselli, "Reliable and Efficient Forwarding in Ad Hoc Networks", vol. 4, pp. 398-415, 2006.

[7] H. Wu and X. Jia, "QoS Multicast Routing by Using Multiple Paths/Trees in Wireless Ad Hoc Networks" Ad Hoc Networks, vol. 5, pp. 600-612, 2009.

[8] R. Braden, D. Clark, and S. Shenker, Integrated Services in the Internet Architecture: An Overview, IETF RFC 1633, 1994.

[9] K. Akkaya, M. Younis, An energy aware QoS routing protocol for wireless sensor networks, in: The Proceedings of the 23rd International Conference on Distributed Computing Systems Workshops, Providence, RI, USA, May, 19-22, 2003, pp.710-715.

[10] I. Stoica and H. Zhang, "Providing Guaranteed Services without Per Flow Management," Proc. ACM Special Interest Group Data Comm. (SIGCOMM), 1999.

[11] P. Jiang, J. Bigham, and J. Wu, "Scalable QoS Provisioning and Service Node Selection in Relay Based Cellular Networks," Proc. Fourth Int'l Conf. Wireless Comm. Networking and Mobile Computing (WiCOM), 2008.

[12] S. Ibrahim, K. Sadek, W. Su, and R. Liu, "Cooperative Communications with Relay-Selection: When to Cooperate and Whom to Cooperate With?" IEEE Trans. Wireless Comm., vol. 7, no. 7, pp. 2814-2827, July 2008.

[13] A. Bletsas, A. Khisti, D.P. Reed, and A. Lippman, "A Simple Cooperative Diversity Method Based on Network Path Selection," IEEE J. Selected Areas in Comm., vol. 24, no. 3, pp. 659-672, Mar. 2006.

[14] T. Ng and W. Yu, "Joint Optimization of Relay Strategies and Resource Allocations in Cellular Networks," IEEE J. Selected Areas in Comm., vol. 25, no. 2, pp. 328-339, Feb. 2004.

[15] J. Cai, X. Shen, J.W. Mark, and A.S. Alfa, "Semi-Distributed User Relaying Algorithm for Amplify-and-Forward Wireless Relay Networks," IEEE Trans. Wireless Comm., vol. 7, no. 4, pp. 13481357, Apr. 2008.

[16] Y. Wei, M. Song, F.R. Yu, Y. Zhang, and J. Song, "Distributed Optimal Relay Selection for QoS Provisioning in Wireless Multihop Cooperative Networks," Proc. IEEE 28th Conf. Global Telecomm. (GlobeCom), pp. 1946-1951, 2009.

[17] Y. Wei and D. Gitlin, "Two-Hop-Relay Architecture for NextGeneration WWAN/WLAN Integration," IEEE Wireless Comm., vol. 11, no. 2, pp. 24-30, Apr. 2004.

[18] C. Lu, B. Blum, T. Abdelzaher, J. Stankovic, and T. He, "RAP: A Real-Time Communication Architecture for Large-Scale Wireless Sensor Networks," Proc. IEEE Real-Time and Embedded Technology Applications Systems, 2002.

[19] T. He, J. Stankovic, C. Lu, and T. Abdelzaher, "SPEED: A Stateless Protocol for Real-Time Communication in Sensor Networks," Proc. 23rd Int'l Conf. Distributed Computing Systems, 2003.

[20] Li, Ze, and Haiying Shen. "QoS-Oriented Distributed Routing Protocol for Hybrid Wireless Networks." (2012): 1-1.

[21] E. Felemban, C. Lee, and E. Ekici, "MMSPEED: Multipath MultiSpeed Protocol for QoS Guarantee of Reliability and Timeliness in Wireless Sensor Networks," IEEE Trans. Mobile Computing, vol. 5, no. 6, pp. 738-754, June 2006.

[22] B. Deb, S. Bhatnagar, and B. Nath, "ReInForm: Reliable Information Forwarding Using Multiple Paths in Sensor Networks," Proc. IEEE 28th Ann. Int'l Conf. Local Computer Networks, 2003. 